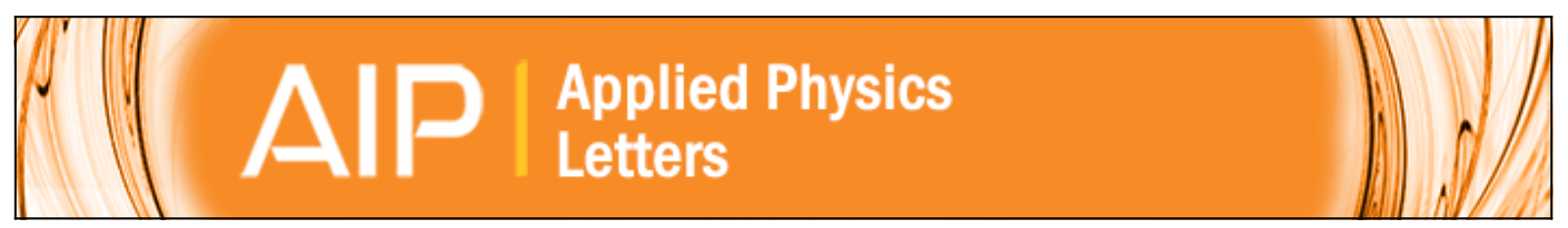

\title{
Helium ion microscope generated nitrogen-vacancy centres in type lb diamond
}

D. McCloskey, D. Fox, N. O'Hara, V. Usov, D. Scanlan, N. McEvoy, G. S. Duesberg, G. L. W. Cross, H. Z. Zhang , and J. F. Donegan

Citation: Applied Physics Letters 104, 031109 (2014); doi: 10.1063/1.4862331

View online: http://dx.doi.org/10.1063/1.4862331

View Table of Contents: http://scitation.aip.org/content/aip/journal/apl/104/3?ver=pdfcov

Published by the AIP Publishing

\section{Articles you may be interested in}

Effect of oxygen plasma and thermal oxidation on shallow nitrogen-vacancy centers in diamond Appl. Phys. Lett. 105, 042406 (2014); 10.1063/1.4891839

Native and induced triplet nitrogen-vacancy centers in nano- and micro-diamonds: Half-field electron paramagnetic resonance fingerprint

Appl. Phys. Lett. 104, 063107 (2014); 10.1063/1.4865205

Diamond nitrogen-vacancy centers created by scanning focused helium ion beam and annealing

Appl. Phys. Lett. 103, 081906 (2013); 10.1063/1.4819339

Depletion of nitrogenvacancy color centers in diamond via hydrogen passivation

Appl. Phys. Lett. 100, 071902 (2012); 10.1063/1.3684612

Engineering of nitrogen-vacancy color centers in high purity diamond by ion implantation and annealing J. Appl. Phys. 109, 083530 (2011); 10.1063/1.3573768

\section{AlP $\mid \begin{aligned} & \text { Journal of } \\ & \text { Applied Physics }\end{aligned}$}

Journal of Applied Physics is pleased to announce André Anders as its new Editor-in-Chief 


\title{
Helium ion microscope generated nitrogen-vacancy centres in type $\mathrm{lb}$ diamond
}

\author{
D. McCloskey, ${ }^{1,2}$ D. Fox,${ }^{1,2}$ N. O'Hara, ${ }^{1,3}$ V. Usov,,${ }^{1,2}$ D. Scanlan, ${ }^{4}$ N. McEvoy, ${ }^{2}$ \\ G. S. Duesberg, ${ }^{2,3}$ G. L. W. Cross, ${ }^{1,2}$ H. Z. Zhang, ${ }^{1,2}$ and J. F. Donegan ${ }^{1,2}$ \\ ${ }^{1}$ School of Physics, Trinity College Dublin, Dublin 2, Ireland \\ ${ }^{2}$ Advanced Materials and Bioengineering Research (AMBER) Centre, CRANN, Trinity College Dublin, \\ Dublin 2, Ireland \\ ${ }^{3}$ School of Chemistry, Trinity College Dublin, Dublin 2, Ireland \\ ${ }^{4}$ Adama Innovations Ltd, The Mews, 61 Serpentine Ave., Dublin 4, Ireland
}

(Received 11 October 2013; accepted 3 January 2014; published online 23 January 2014)

\begin{abstract}
We report on position and density control of nitrogen-vacancy (NV) centres created in type Ib diamond using localised exposure from a helium ion microscope and subsequent annealing. Spatial control to $<380 \mathrm{~nm}$ has been achieved. We show that the fluorescence lifetime of the created centres decreases with increasing ion dose. Furthermore, we show that for doses $>1 \times 10^{17}$ ion/ $/ \mathrm{cm}^{2}$, significant damage of the diamond lattice occurs resulting in fluorescence quenching and amorphization. This places an upper limit on the density of NV centres that can be created using this method. (C) 2014 AIP Publishing LLC. [http://dx.doi.org/10.1063/1.4862331]
\end{abstract}

The nitrogen-vacancy centre (NV) in diamond is a wellstudied, optically active colour centre formed by a substitutional nitrogen atom and a nearest-neighbour lattice vacancy. These centres are naturally present in low concentration in diamond containing nitrogen and have received considerable attention recently for their spin properties, ${ }^{1}$ room temperature photo-stability, ${ }^{2}$ potential to create single photon sources, ${ }^{3}$ use in biological sensing, ${ }^{4}$ and as tools for nanoscopy ${ }^{5}$ and magnetometry. ${ }^{6}$

For many of these applications, precise position and density control of colour centres is essential. For example, the emission rate and collection efficiency from a single NV centre can be significantly enhanced by coupling to photonic resonators. ${ }^{7}$ The degree of this enhancement (Purcell effect) depends greatly on optimising the position of the centre with respect to the spatial distribution of the resonator mode, which requires nanoscale precision. ${ }^{8}$ Enhancements of the photon emission rate up to $\sim 12$ times have been achieved experimentally by coupling to a photonic crystal cavity. ${ }^{9}$ Using AFM techniques to manipulate nanocrystals of diamond containing a single NV centre allows nanometer scale position control. ${ }^{10}$ For applications based on spin coupling in large groups of NV centres, position control and density control are also important. NV centres coupled to superconducting resonators can be employed as qubits, the single elements for building quantum computers. ${ }^{11}$

$\mathrm{NV}$ centres in diamond can be produced by irradiation with electrons, neutrons, or high energy ions and subsequent annealing. The irradiation creates vacancies in the diamond lattice, which diffuse under annealing. These are gettered by nitrogen impurities present in the diamond to form the NV centre. Two charge states of this centre exist, labelled $\mathrm{NV}^{0}$ and $\mathrm{NV}^{-}$, which have zero phonon lines (ZPL) at $575 \mathrm{~nm}$ and $638 \mathrm{~nm}$, respectively. The $\mathrm{NV}^{-}$centre has the optical and spin properties required for quantum information processing and magnetic sensing and thus is preferable to the $\mathrm{NV}^{0}$ centre. ${ }^{12}$ Electron irradiation provides position control; however, it greatly reduces the intensity of the $\mathrm{NV}^{-}$centre due to its annihilation with radiation-induced interstitials. ${ }^{13}$ Similarly, neutron irradiation suppresses the creation of $\mathrm{NV}^{-}$ centres by conversion of the $\mathrm{NV}^{-}$to $\mathrm{NV}^{0} .{ }^{14} \mathrm{He}^{+}$irradiation has been shown as a promising method to efficiently create $\mathrm{NV}^{-}$centres in crystalline diamond. ${ }^{15}$ High energy $(\sim 2-3 \mathrm{MeV}) \mathrm{He}^{+}$implantation requires particle accelerators. ${ }^{16}$ These techniques typically produce doses of $10^{17}$ ions $\mathrm{cm}^{-2}$ and are limited to $>5 \times 10^{13}$ ions $\mathrm{cm}^{-2}$. High energy $\mathrm{He}^{+}$irradiation can be combined with masking techniques to gain spatial control on the order of tens of microns; however, $\mathrm{He}^{+}$scattering from the mask causes unwanted vacancies up to $60 \mu \mathrm{m}$ from the desired position. ${ }^{16}$

Recently, low energy $(\sim 40 \mathrm{keV}) \mathrm{He}^{+}$ions generated by radio frequency ion sources methods have been shown to efficiently create NV centres in nanodiamond. ${ }^{4}$ Furthermore, lower energy $\mathrm{He}^{+}$ions have a larger interaction cross section and can create 40 vacancies per ion at $40 \mathrm{keV}$ compared to 13 for $3 \mathrm{MeV} \mathrm{H}^{+}$and 0.1 for $2 \mathrm{MeV} \mathrm{e}^{-}$, respectively. ${ }^{17}$ This efficiency allows use of a much smaller ion dose compared to high energy irradiation. In order to achieve nanometer scale spatial positioning precision of NV centres, techniques employing focused ion beams can be used. Focused gallium ions at $30 \mathrm{keV}$ can create $\mathrm{NV}$ centres localized to a $\sim 180 \mathrm{~nm}$ lateral extent with a distribution centred $15 \mathrm{~nm}$ from the top surface. ${ }^{18}$ Single NV centres in type IIa diamond can be created by focused nitrogen implantation with a $2.5 \%$ conversion efficiency. ${ }^{19}$ Recently, Huang et al. reported creation of $\mathrm{NV}$ centres in diamond using a He-ion microscope. ${ }^{20}$ This technique combines the advantage of low energy $\mathrm{He}^{+}$ion irradiation with the spatial control of focused ion beam techniques. Subsequent annealing creates NV centres. Spatial control is achieved through raster scanning of the beam, eliminating problems of ion scattering from a mask. They show that NV centres can be created with a $10 \%$ conversion efficiency and place an upper limit on their spatial distribution of $600 \mathrm{~nm}^{20}$

Here, we use a helium ion microscope (HIM) to irradiate type $\mathrm{Ib}\langle 100\rangle$ orientation diamond with nitrogen content $>200 \mathrm{ppm}$ and study the optical properties of the centres we 
create. Spatial and spectral mapping allows us to show that the $\mathrm{NV}^{0}$ and $\mathrm{NV}^{-}$centres have the same distribution within the irradiated areas. The density of NV centres is shown to vary linearly as a function of He-ion dose. Spatial control below the diffraction limit of the collection objective is demonstrated by fitting a sharp edge with the blurred edge function. Fluorescence lifetime imaging microscopy (FLIM) shows a two component fluorescence lifetime ( $2 \mathrm{~ns}$ and $8 \mathrm{~ns}$ ) similar to results from NV centres created from neutron irradiation. ${ }^{21}$ The lifetime is shown to decrease with increasing ion dose, which we attribute to increasing damage in the crystal causing non-radiative decay paths. We show that for doses of $10^{17}$ ions $/ \mathrm{cm}^{2}$ and above, NV centre fluorescence is quenched due to amorphization of the diamond.

The HIM is a recently developed tool which enables sub-nanometre resolution imaging, as well as nanoscale modification of materials. The HIM has a demonstrated capability to reliably and reproducibly introduce a tuneable density of defects into materials. ${ }^{22}$ Samples of single crystal $\langle 100\rangle$ type $\mathrm{Ib}$ diamond were chosen due to the abundance of single substitutional nitrogen defects (P1 centers) in such materials. The nitrogen doping was sufficient to give the sample a yellow appearance resulting from strong absorption around $400 \mathrm{~nm}$ due to the P1 centers (Fig. 1(a)). Raman spectra show a strong first order peak at $1332 \mathrm{~cm}^{-1}$ with no other observable peaks such as those from amorphous carbon, confirming the high quality of the diamond sample ${ }^{23}$ (Fig. 1(b)). The nitrogen content of the diamond was calculated to be $246 \mathrm{ppm}$ from the ratio of the $1130 \mathrm{~cm}^{-1} \mathrm{P} 1$ center peak in the FTIR spectrum to the background lattice absorption dip at $2120 \mathrm{~cm}^{-1}$ (Refs. 24 and 25) (Fig. 1(c)).

The diamond samples were irradiated with a focused beam of $30 \mathrm{keV}$ helium ions using the HIM. The $\mathrm{He}^{+}$ions were directed with an incident angle of $2^{\circ}$ with respect to the normal of the sample surface to avoid ion channeling. The ion distribution, shown in Fig. 1(d), was calculated using Monte Carlo simulations (The Stopping and Range of ions in matter-SRIM). The projection onto the $y-z$ plane shows the number density a maximum of number density of $16 \times 10^{4} \mathrm{~cm}^{-1}$. The spatial density distribution of vacancies from a single spot exposure is shown in Fig. 1(e). A single $30 \mathrm{keV} \mathrm{He}{ }^{+}$will create on average 67 carbon displacements with 4 replacement collisions resulting in a total of 63 vacancies. This provides a high yield of localized vacancies. The projection on the $\mathrm{y}-\mathrm{z}$ axis shows a maximum of 0.06 displacements per angstrom per ion. The vacancies are expected to be concentrated $130 \pm 30 \mathrm{~nm}$ from the surface with a lateral spread of $\sim 40 \mathrm{~nm}$. To form the NV centers, samples were placed in a carbolite tube furnace and ramped to $800^{\circ} \mathrm{C}$ at a rate of $400{ }^{\circ} \mathrm{C} / \mathrm{h}$ under Ar flow at a pressure of $1.3 \mathrm{mbar}$. The samples were held at this temperature for $1 \mathrm{~h}$ to allow vacancy diffusion and then cooled to room temperature under Ar flow before venting.

Photoluminescence (PL) measurements we conducted using a $488 \mathrm{~nm} \mathrm{CW}$ excitation laser with $1 \mathrm{~mW}$ excitation power. Collected light was passed through a dichroic mirror and analyzed using a Reinshaw in Via spectrometer to allow a full spectrum to be collected at each point in space. The sample was raster scanned using a calibrated piezoelectric positioning stage. Figure 1(f) shows the integrated PL
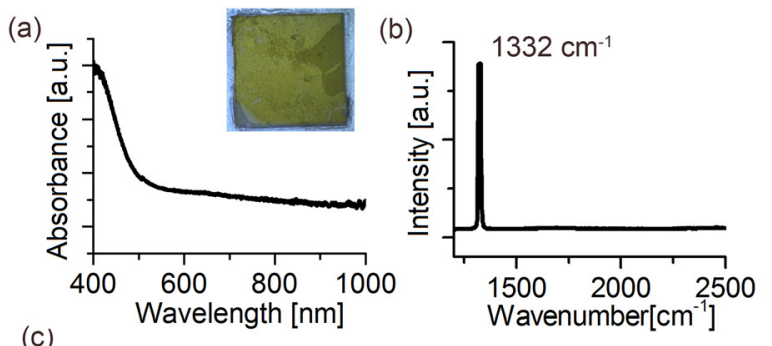

(c)
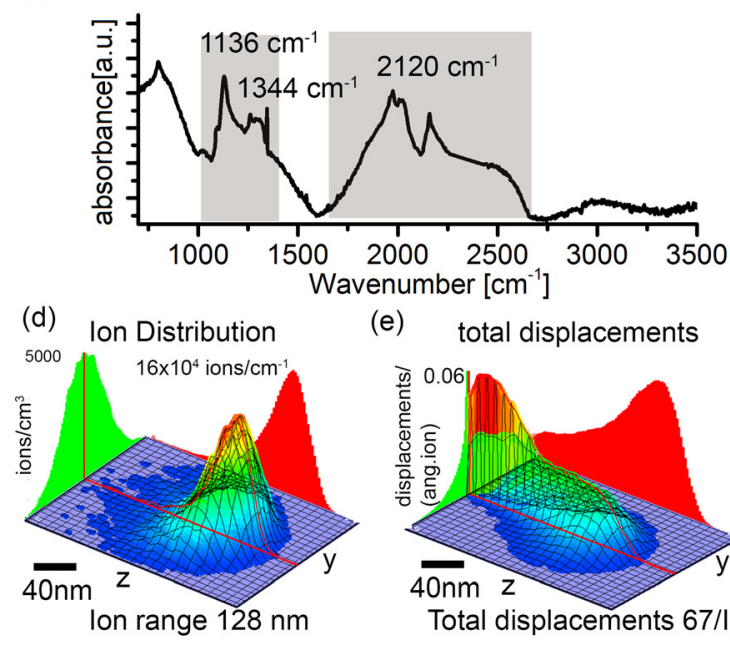

(e) total displacements
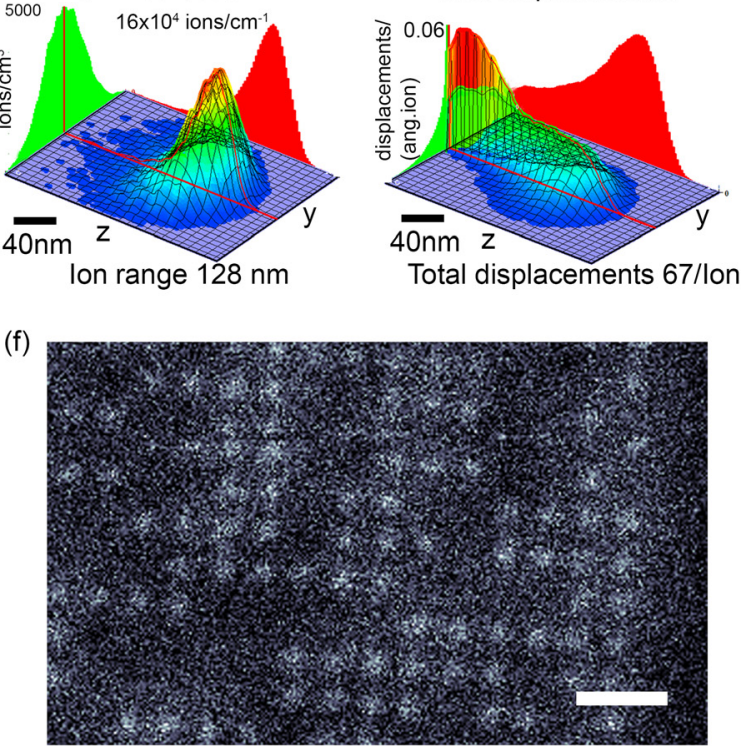

FIG. 1. (a) Optical absorbance spectrum of type Ib diamond. Peak at $400 \mathrm{~nm}$ due to single substitutional nitrogen (P1 centre). Inset shows optical micrograph of $3 \mathrm{~mm} \times 3 \mathrm{~mm}$ sample. (b) Raman spectrum showing peak at $1333 \mathrm{~cm}^{-1}$. (c) FTIR spectrum with peaks at $1136 \mathrm{~cm}^{-1}$ and $1344 \mathrm{~cm}^{-1}$ due to $\mathrm{P} 1$ centres, with additional structure from $1600 \mathrm{~cm}^{-1}$ to $2600 \mathrm{~cm}^{-1}$ due to self-absorption of the diamond lattice. (d) SRIM simulation of the distribution of $\mathrm{He}$ ions with $30 \mathrm{keV}$ exposure. 2D plots show number density of ions in units of $\mathrm{cm}^{-1}$. (e) SRIM simulation of carbon displacements within diamond under same conditions. 2D plots show displacements per angstrom per ion. (f) Spatial PL map of random array of spot exposures after annealing using $1 \times 10^{13}$ ions $/ \mathrm{cm}^{2}$ illustrating diffraction limited localisation of $\mathrm{NV}$ centres. Scale bar $2 \mu \mathrm{m}$.

intensity over a $4 \mathrm{~nm}$ bandwidth in centered at the $637 \mathrm{~nm}$ $\mathrm{ZPL}$ of the $\mathrm{NV}^{-}$center for a patterned array of point exposures. The spot exposures were made with $1 \mu \mathrm{m}$ separation using $30 \mathrm{keV}$ acceleration voltage, $0.8 \mathrm{pA}$ beam current and $20 \mathrm{~ms}$ dwell time, resulting in a dose of $1 \times 10^{13}$ ions $/ \mathrm{cm}^{2}$. The patterning for instance, shows that these points could be used to store information such as a barcode.

To study the effect of ion dose quantitatively arrays of $5 \mu \mathrm{m} \times 5 \mu \mathrm{m}$ squares were patterned with $10 \mu \mathrm{m}$ separation. The area was defined by raster scanning the He-ion beam in $10 \mathrm{~nm}$ step sizes, with a beam current of $0.8 \mathrm{pA}$ and the average dose controlled by varying the dwell time. The samples were annealed as described previously and a spatial map of PL intensity from the $637 \mathrm{~nm}$ ZPL is shown in Fig. 2(a). 
(a)

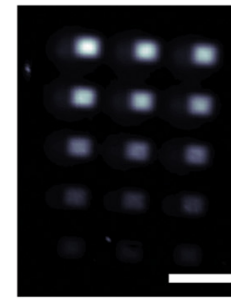

(d)

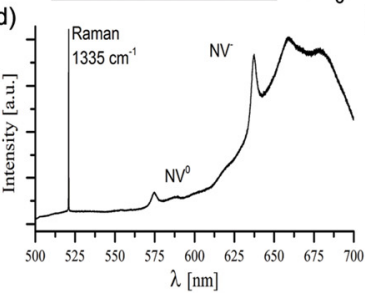

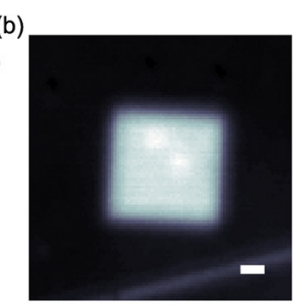

(e)

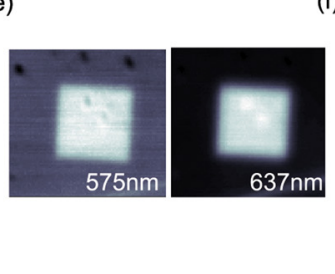

(c)

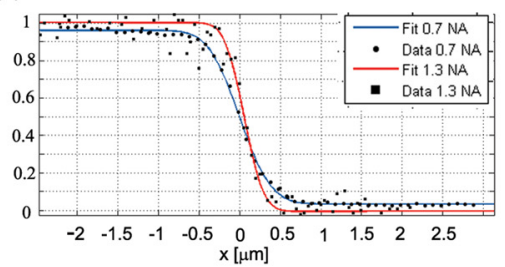

(f)

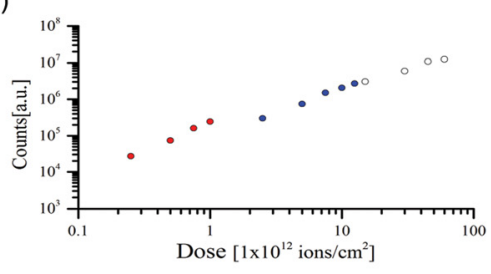

FIG. 2. (a) PL scans of $5 \mu \mathrm{m} \times 5 \mu \mathrm{m}$ exposed areas. Continuous wave excitation from $\mathrm{Ar}^{+}$at $488 \mathrm{~nm}$ with $1 \mathrm{~mW}$ power was used. Colour map is in counts integrated over a $4 \mathrm{~nm}$ bandwidth around the $637 \mathrm{~nm}$ ZPL. No conversion is made to counts per second. Each row is a different dose with 3 repeats. The doses are $12.5 \times 10^{12}$ ions $/ \mathrm{cm}^{2}, 10 \times 10^{12}$ ions $/ \mathrm{cm}^{2}, 7.5 \times 10^{12}$ ions $/ \mathrm{cm}^{2}, 5 \times 10^{12}$ ions $/ \mathrm{cm}^{2}$, and $2.5 \times 10^{12}$ ions $/ \mathrm{cm}^{2}$, from top to bottom, respectively. (b) High resolution scan of a single exposed $5 \mu \mathrm{m} \times 5 \mu \mathrm{m}$ area using $100 \times$ objective with NA $=0.7$. The scan area is $10 \mu \mathrm{m} \times 10 \mu \mathrm{m}$, with step size of $100 \mathrm{~nm}$. (c) Experimental line section over the edge of the exposed area fitted with a blurred edge function. Taken with NA $=0.7$ and NA $=1.3$ objectives. (d) Extended scan of the fluorescence from $480 \mathrm{~nm}$ to $750 \mathrm{~nm}$. The $\mathrm{NV}^{0}$ and NV $\mathrm{NV}^{-}$lines are observed and also fluorescence from the vibronic sidebands associated with these defects. The fluorescence is strong compared with the Raman peak. (e) Comparison between the spatial distribution of PL intensity averaged over $4 \mathrm{~nm}$ bandwidth on the $575 \mathrm{~nm} \mathrm{NV}^{0} \mathrm{ZPL}$ and the $637 \mathrm{~nm} \mathrm{NV}^{-} \mathrm{ZPL}$, respectively. This shows that both centres are created uniformly. (f) Linear dependence of PL counts from the $637 \mathrm{~nm}$ ZPL as a function of He-ion dose. Three separate arrays similar to Fig. 2(a) were measured (red, blue, and white dots).

Each row of the array repeats the same dose 3 times, starting from $12.5 \times 10^{12}$ ions $/ \mathrm{cm}^{2}$, and decreasing to $10 \times 10^{12}$ ions $/ \mathrm{cm}^{2}, \quad 7.5 \times 10^{12}$ ions $/ \mathrm{cm}^{2}, \quad 5 \times 10^{12}$ ions $/ \mathrm{cm}^{2}$, and $2.5 \times 10^{12}$ ions $/ \mathrm{cm}^{2}$, from top to bottom, respectively. The minimum beam current and dwell time of the tool set a lower limit on the dose of $6.3 \times 10^{10}$ ions $/ \mathrm{cm}^{2}$ (when using a $10 \mathrm{~nm}$ step size). In addition to the array of Fig. 2(a), two separate arrays were patterned covering the dose range from $2.5 \times 10^{11}$ ions $/ \mathrm{cm}^{2}$ to $6 \times 10^{13}$ ions $/ \mathrm{cm}^{2}$. The integrated PL intensity of the $\mathrm{NV}^{-}$ZPL from these regions is plotted as a function of dose in Fig. 2(f). This intensity varies linearly over the full variation of dose, indicating that one nitrogen atom is used per defect.

The room temperature PL spectrum from $500 \mathrm{~nm}$ to $700 \mathrm{~nm}$ is shown in Fig. 2(d). The $\mathrm{NV}^{0} \mathrm{ZPL}$ at $575 \mathrm{~nm}, \mathrm{NV}^{-}$ ZPL at $637 \mathrm{~nm}$, and phonon side bands are clearly observed. Spatial maps of the PL intensity with $4 \mathrm{~nm}$ band-pass centred on the $575 \mathrm{~nm}$ ZPL and the $637 \mathrm{~nm}$ ZPL show that both centres are evenly distributed throughout the sample within the limit of the optical resolution of the microscope (Fig. 2(e)).

These results show that the NV centres can be created with high spatial accuracy. A high resolution spatial PL map of a single exposed $5 \mu \mathrm{m} \times 5 \mu \mathrm{m}$ area is shown in Fig. 2(b). To confirm the spatial localisation of the NV centres, a line profile can be taken across the edge of this exposure and compared to the expected blurring from the point spread function (PSF) of the microscope objective. ${ }^{26}$ Figure 2(c) shows the data and fits for an objective with a numerical aperture (NA) 0.7 (blue) and an oil immersion objective with NA 1.3 (red). Fitting shows that NV centres can be created with a spatial extent $<380 \mathrm{~nm}$.

The lifetime of the NV centre fluorescence in bulk diamond is approximately referred to as $\sim 12 \mathrm{~ns}$. This single exponential lifetime was originally measured to be $13 \pm 0.5 \mathrm{~ns}$ by Collins et al. in 1983 using a flash lamp. ${ }^{27}$ Since then the lifetime has been measured using a picosecond excitation source in NV centres in type Ib diamond formed by high energy neutron irradiation. It was found that this $12 \mathrm{~ns}$ decay was in fact a bi-exponential decay with short component $2 \mathrm{~ns}$ and long component $8 \mathrm{~ns}^{21}$ This shorter bi-exponential decay was not explained and was thought to possibly originate from damage of the lattice from the high energy exposure. Here, we show that the NV centres produced using low energy He-ion irradiation also have a bi-exponential behaviour with short component of $2 \mathrm{~ns}$ and long component of $8 \mathrm{~ns}$. A commercial FLIM system (Microtime 200, PicoQuant) was used to measure the fluorescence lifetimes at different positions in the sample. A $466 \mathrm{~nm}$ picosecond laser with a pulse FWHM of $63 \mathrm{ps}$, repetition rate of $10 \mathrm{MHz}$, and average power of $0.9 \mathrm{~mW}$ was used. The lifetime was found to decrease with increasing $\mathrm{He}^{+}$dose. Figure 3(a) shows a spatial map of the lifetimes an array of four $15 \mu \mathrm{m} \times 15 \mu \mathrm{m}$ areas of $\mathrm{NV}$ centres with doses $10^{13}, 10^{14}, 10^{15}$, and $10^{16}$ ions $/ \mathrm{cm}^{2}$. The normalised fluorescence decays for each dose are shown on a semi-log scale in Fig. 3(b). The tails are well fitted with a bi-exponential (solid line), with time constants given in the figure.

Finally, it was noted that for doses $10^{17}$ ions $/ \mathrm{cm}^{2}$ and above the diamond was blackened allowing the exposed area to be visible under the optical microscope. A fluorescence map of a $5 \mu \mathrm{m} \times 5 \mu \mathrm{m}$ area exposed to $10^{17} \mathrm{ions} / \mathrm{cm}^{2}$ is shown in Fig. 4(a). Fluorescence is quenched in the central region and remains in surrounding regions which obtained a lower effective ion dose. Comparative Raman spectra at positions just outside (1) and inside (2) the directly exposed region show that where the fluorescence is missing a significant amount of the diamond has been converted to amorphous carbon. The $\mathrm{D}$ and $\mathrm{G}$ bands of amorphous carbon, along with the Raman peak from diamond lattice in the focal volume are clearly observed at position 2 .

We have demonstrated a technique to create NV centres in diamond with position and density control. We show that 
(a)
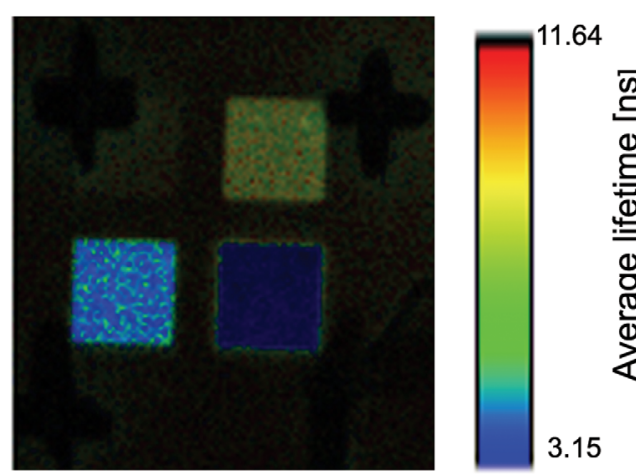

(b)

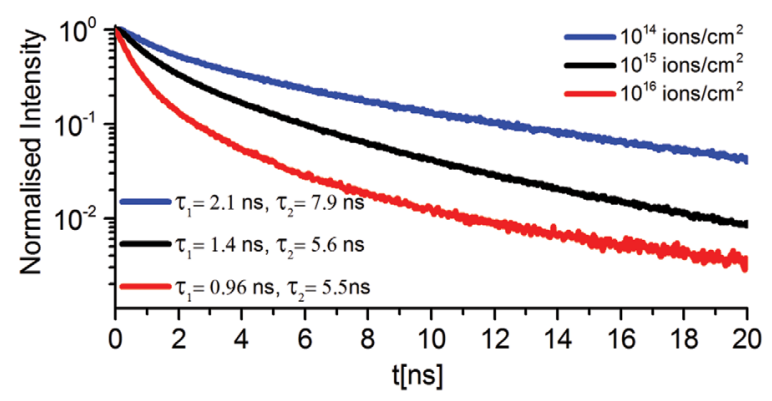

FIG. 3. (a) Fluorescence lifetime map of a $60 \mu \mathrm{m} \times 60 \mu \mathrm{m}$ area with $15 \mu \mathrm{m} \times 15 \mu \mathrm{m}$ areas of NV centers. The doses used were: top left: $10^{13}$ ions $/ \mathrm{cm}^{2}$, top right: $10^{14}$ ions $/ \mathrm{cm}^{2}$, bottom left: $10^{15}$ ions $/ \mathrm{cm}^{2}$, bottom right $10^{16}$ ions $/ \mathrm{cm}^{2}$. (b) Normalised fluorescence decays after picosecond excitation. The measured lifetime decreases with increasing dose. (a)

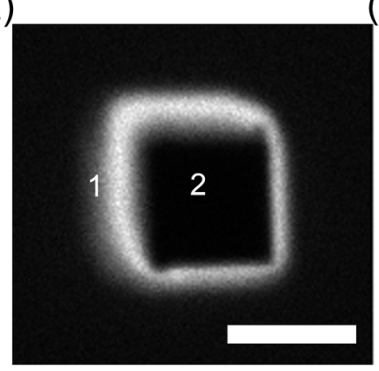

(b)

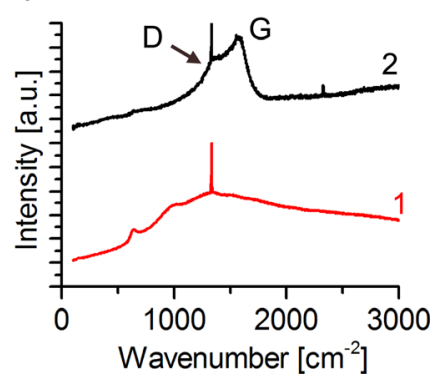

FIG. 4. (a) Fluorescence map of a $5 \mu \mathrm{m} \times 5 \mu \mathrm{m}$ area exposed to $10^{17}$ ions $/ \mathrm{cm}^{2}$. Fluorescence is quenched in the central region (b) Raman spectra at positions 1 and 2 shown in (a). Position 1 has a fluorescence background with a strong Raman peak at $1332 \mathrm{~cm}^{-1}$ due to the diamond lattice. Position 2 shows no PL but the D and G bands of amorphous carbon, along with the Raman peak from diamond lattice in the focal volume.

the position can be controlled to within $<380 \mathrm{~nm}$. Spectrally resolved spatial mapping of the fluorescence shows a homogeneous distribution of $\mathrm{NV}^{0}$ and $\mathrm{NV}^{-}$centres are created. FLIM studies show that these centres have a bi-exponential fluorescence decay similar to NV centres created by high energy proton and neutron irradiation. ${ }^{24}$ The decay time was shown decrease with increasing ion dose. This effect was attributed to increased damage in the crystal lattice providing non radiative decay paths. ${ }^{28}$ For doses larger than $10^{17}$ ions $/ \mathrm{cm}^{2}$, the exposed areas became dark due to amorphization of the diamond lattice confirmed using Raman spectroscopy. This sets an upper limit on the usable dose to create ensembles of NV centres with this method. This technique can be used to control the position and number density of NV centres for applications in biological sensing, encryption, quantum computing, and magnetometry. Using higher doses allows controlled amorphization of diamond with nanoscale precision, which could be used for nanofabrication.

This publication has emanated from research supported in part by a research grant from Science Foundation Ireland (SFI) under Grant No. SFI/12/RC/2278.

${ }^{1}$ T. Gaebel, M. Domhan, I. Popa, C. Wittmann, P. Neumann, F. Jelezko, J. R. Rabeau, N. Stavrias, A. D. Greentree, S. Prawer, J. Meijer, J. Twamley, P. R. Hemmer, and J. Wrachtrup, Nat. Phys. 2(6), 408 (2006).

${ }^{2}$ A. Gruber, A. Dräbenstedt, C. Tietz, L. Fleury, J. Wrachtrup, and C. von Borczyskowski, Science 276(5321), 2012 (1997).

${ }^{3}$ T. M. Babinec, J. M. HausmannBirgit, M. Khan, Y. Zhang, J. R. Maze, P. R. Hemmer, and M. Loncar, Nat. Nanotechnol. 5(3), 195 (2010).

${ }^{4}$ Y. Chang, H. Lee, K. Chen, C. Chang, D. Tsai, C. Fu, T. Lim, Y. Tzeng, C. Fang, C. Han, H. Chang, and W. Fann, Nat. Nanotechnol. 3(5), 284 (2008).

${ }^{5}$ I. Aharonovich, A. D. Greentree, and S. Prawer, Nat. Photonics 5(7), 397 (2011).

${ }^{6}$ G. Balasubramanian, I. Y. Chan, R. Kolesov, M. Al-Hmoud, J. Tisler, C. Shin, C. Kim, A. Wojcik, P. R. Hemmer, A. Krueger, T. Hanke, A. Leitenstorfer, R. Bratschitsch, F. Jelezko, and J. Wrachtrup, Nature 455(7213), 648 (2008); S. Steinert, F. Dolde, P. Neumann, A. Aird, B. Naydenov, G. Balasubramanian, F. Jelezko, and J. Wrachtrup, Rev. Sci. Instrum. 81(4), 043705 (2010); J. R. Maze, P. L. Stanwix, J. S. Hodges, S. Hong, J. M. Taylor, P. Cappellaro, L. Jiang, M. V. Gurudev Dutt, E. Togan, A. S. Zibrov, A. Yacoby, R. L. Walsworth, and M. D. Lukin, Nature 455(7213), 644 (2008).

${ }^{7}$ A. Faraon, P. E. Barclay, C. Santori, K. C. Fu, and R. G. Beausoleil, Nat. Photonics 5(5), 301 (2011); K. J. Vahala, Nature 424(6950), 839 (2003).

${ }^{8}$ J. M. Gérard, B. Sermage, B. Gayral, B. Legrand, E. Costard, and V. Thierry-Mieg, Phys. Rev. Lett. 81(5), 1110 (1998).

${ }^{9}$ J. Wolters, A. W. Schell, G. Kewes, N. Nusse, M. Schoengen, H. Doscher, T. Hannappel, B. Lochel, M. Barth, and O. Benson, Appl. Phys. Lett. 97(14), 141108 (2010).

${ }^{10}$ T. van der Sar, E. C. Heeres, G. M. Dmochowski, G. de Lange, L. Robledo, T. H. Oosterkamp, and R. Hanson, Appl. Phys. Lett. 94(17), 173104 (2009); P. E. Barclay, C. Santori, K. Fu, R. G. Beausoleil, and O. Painter, Opt. Express 17(10), 8081 (2009); O. Benson, Nature 480(7376), 193 (2011).

${ }^{11}$ A. Imamoğlu, Phys. Rev. Lett. 102(8), 083602 (2009); Y. Kubo, F. R. Ong, P. Bertet, D. Vion, V. Jacques, D. Zheng, A. Dréau, J. F. Roch, A. Auffeves, F. Jelezko, J. Wrachtrup, M. F. Barthe, P. Bergonzo, and D. Esteve, ibid. 105(14), 140502 (2010).

${ }^{12}$ F. Jelezko and J. Wrachtrup, Phys. Status Solidi A 203(13), 3207 (2006).

${ }^{13}$ J. W. Steeds, T. J. Davis, S. J. Charles, J. M. Hayes, and J. E. Butler, Diamond Relat. Mater. 8(10), 1847 (1999).

${ }^{14}$ Y. Mita, Phys. Rev. B 53(17), 11360 (1996).

${ }^{15}$ F. C. Waldermann, P. Olivero, J. Nunn, K. Surmacz, Z. Y. Wang, D. Jaksch, R. A. Taylor, I. A. Walmsley, M. Draganski, P. Reichart, A. D. Greentree, D. N. Jamieson, and S. Prawer, Diamond Relat. Mater. 16(11), 1887 (2007).

${ }^{16}$ J. O. Orwa, K. Ganesan, J. Newnham, C. Santori, P. Barclay, K. M. C. Fu, R. G. Beausoleil, I. Aharonovich, B. A. Fairchild, P. Olivero, A. D. Greentree, and S. Prawer, Diamond Relat. Mater. 24(0), 6 (2012).

${ }^{17}$ T. Wee, Y. Tzeng, C. Han, H. Chang, W. Fann, J. Hsu, K. Chen, and Y. Yu, J. Phys. Chem. A 111(38), 9379 (2007); S. C. Lawson, D. Fisher, D. C. Hunt, and M. E. Newton, J. Phys.: Condens. Matter 10(27), 6171 (1998).

${ }^{18}$ J. Martin, R. Wannemacher, J. Teichert, L. Bischoff, and B. Kohler, Appl. Phys. Lett. 75(20), 3096 (1999).

${ }^{19}$ J. Meijer, B. Burchard, M. Domhan, C. Wittmann, T. Gaebel, I. Popa, F. Jelezko, and J. Wrachtrup, Appl. Phys. Lett. 87(26), 261909 (2005).

${ }^{20}$ Z. Huang, W.-D. Li, C. Santori, V. M. Acosta, A. Faraon, T. Ishikawa, W. Wu, D. Winston, R. S. Williams, and R. G. Beausoleil, Appl. Phys. Lett. 103(8), 081906 (2013).

${ }^{21}$ H. Hanzawa, Y. Nisida, and T. Kato, Diamond Relat. Mater. 6(11), 1595 (1997).

${ }^{22}$ D. Fox, Y. B. Zhou, A. O'Neill, S. Kumar, J. J. Wang, J. N. Coleman, G. S. Duesberg, J. F. Donegan, and H. Z. Zhang, Nanotechnology 24(33), 335702 (2013). 
${ }^{23}$ S. A. Solin and A. K. Ramdas, Phys. Rev. B 1(4), 1687 (1970).

${ }^{24}$ L. Su, C. Fang, Y. T. Chang, K. M. Chen, Y. C. Yu, J. H. Hsu, and H. C. Chang, Nanotechnology 24(31), 315702 (2013).

${ }^{25}$ Z. Z. Liang, X. Jia, H. A. Ma, C. Y. Zang, P. W. Zhu, Q. F. Guan, and H. Kanda, Diamond Relat. Mater. 14(11-12), 1932 (2005).
${ }^{26} \mathrm{M}$. Hagara and P. Kulla, Radioengineering 20(2), 516 (2011).

${ }^{27}$ A. T. Collins, M. F. Thomaz, and M. I. B. Jorge, J. Phys. C: Solid State Phys. 16, 2177 (1983).

${ }^{28}$ J. O. Orwa, K. W. Nugent, D. N. Jamieson, and S. Prawer, Phys. Rev. B 62(9), $5461(2000)$. 